\title{
Energy Constrained Sandpile Models
}

\author{
Alessandro Chessa, ${ }^{1,2}$ Enzo Marinari, ${ }^{1,3}$ and Alessandro Vespignani ${ }^{4}$ \\ ${ }^{1}$ Dipartimento di Fisica, Università di Cagliari, Via Ospedale 72, 09124 Cagliari, Italy \\ ${ }^{2}$ Istituto Nazionale di Fisica della Materia (INFM), Sezione di Cagliari, Italy \\ ${ }^{3}$ Istituto Nazionale di Fisica Nucleare (INFN), Sezione di Cagliari, Italy \\ ${ }^{4}$ International Center for Theoretical Physics (ICTP), P.O. Box 586, 34100 Trieste, Italy
}

(Received 11 December 1997)

\begin{abstract}
We study two driven dynamical systems with conserved energy. The two automata contain the basic dynamical rules of the Bak, Tang, and Wiesenfeld sandpile model. In addition a global constraint on the energy contained in the lattice is imposed. In the limit of an infinitely slow driving of the system, the conserved energy $E$ becomes the only parameter governing the dynamical behavior of the system. Both models show scale-free behavior at a critical value $E_{c}$ of the fixed energy. The scaling with respect to the relevant scaling field points out that the developing of critical correlations is in a different universality class than self-organized critical sandpiles. Despite this difference, the activity (avalanche) probability distributions appear to coincide with the one of the standard self-organized critical sandpile. [S0031-9007(98)06024-4]
\end{abstract}

PACS numbers: 64.60.Lx, 05.40.+j, 05.70.Ln, 46.10.+z

In the study of nonequilibrium critical phenomena, cellular automata (CA) showing self-organized criticality (SOC) have acquired a very special role [1,2]. Differently from the usual continuous phase transitions, they would spontaneously evolve into a critical state without explicit fine tuning of control parameters. Another reason of interest lies in the fact that numerical computations based on SOC ideas have shown that slowly driven systems can lead to a stationary state with a dynamical activity characterized by avalanches of widely distributed amplitude [2]. Avalanche behavior is a basic feature of many experimentally observed phenomena ranging from magnetic systems [3] to microfracturing process [4] and earthquakes [5]. The prototypical model for SOC is represented by Bak, Tang, and Wiesenfeld (BTW) sandpile automata [1], in which an infinitesimally slow external driving of sand particles associated with a threshold rearrangement dynamics leads to a stationary state with activity (avalanches) distributed on all length scales [1]. More widely, the model is generalized by identifying the sand grain as energy, stress, or pressure quanta. In this way the analogy with other physical phenomena appears more clearly.

Despite the vast activity in the field, the general picture of SOC phenomena contains many ambiguities. It has been pointed out by several authors [2,6,7] that the driving rate acts exactly as a control parameter that has to be fine tuned to zero in order to observe criticality. For instance, in sandpile the stationary state results from the balance of the driving field and dissipation rates intrinsically operating because of the system open boundary. The critical point is reached only through a limit process in which both driving and dissipation rates tend to zero. This point corresponds to a locality breaking of the dynamical rules [7] that determines the onset of the critical correlation properties [8]. In this framework many relations with nonequilibrium critical phenomena, such as adsorbing critical point [9], have been enlightened. However, many important issues are still open. It is not clear the interplay among the self-organization into a stationary state due to the energy balance and the dynamical developing of correlations. Also numerically many important features, such as critical exponents, universality classes, and the upper critical dimension are very difficult to obtain to a sufficient degree of precision $[10,11]$. This is mainly due to the inherent strong effect of finite size corrections present in the original model; the boundary size plays an active role, being the only dissipative ingredient leading to the stationary state [7].

In this Letter we introduce a stochastic CA which contains the basic elements of the sandpile model, but is defined on a lattice with periodic boundary conditions, and has a global constraint in the energy accumulated. The average energy contained in the system is therefore constant and fixed from the outside. This resembles a microcanonical definition of the sandpile automata. The reason for studying this model is twofold: First, it seems more appropriate to represent some phenomena in which the dissipation is not intrinsically linked to the activity of the systems. Second, it could shed light on many properties of the SOC sandpile by allowing its analysis in a framework which looks closer to the usual statistical physics. Finally, it turns out that microcanonical sandpiles do not suffer heavily of finite-size correction effects because of the possibility of using effectively periodic boundary conditions. Thus, the microcanonical sandpile could be used to settle universality classes and upper critical dimension issues.

We consider two models with conserved energy. In both we start from a given sand configuration $\left\{e_{i}\right\}$, that can be random or the result of a former run (if needed after modifying its energy), where $i=(x, y)$ labels the $L^{2}$ sites of a $2-d$ lattice with periodic boundary conditions. 
The total amount of sand (the energy of the system) is $E \equiv \sum_{i} e_{i}$. The system is supposed to be in contact with an external reservoir with which it can exchange energy in both directions; an incoming as well as an outcoming energy flux is present. We think of the system as in a sort of thermal equilibrium with a fixed value of energy. This implies the two fluxes on average are equal.

In both models, the first stage of the dynamics is the addition of an energy unit on a random site. In order to preserve the total amount of energy, we have to introduce an extraction flux that takes away one unit of energy from the system. We do that in two ways. In the first model (that we call random subtraction, RS) we take away one unit of energy in a random site: this model is discrete, and $e_{i}$ can only take integer values from 0 to 4 . In the second model (with a continuous subtraction, CS) we have a homogeneous dissipation, where each site looses energy proportionally to the local energy density. Here we transform $e_{i} \rightarrow e_{i} \frac{E}{E+1}$. The basic variables of this second model are continuous, and can take values between 0 and 4.

The internal dynamics of both models is supposed to be very fast with respect to the energy addition and extraction rates, in analogy with the slow driving assumption commonly used in SOC models. After the energy addition and extraction, the avalanching process follows in the usual way. If $e_{i}$ is larger or equal to 4 (the critical threshold for local stability), the energy on the site gets shared among the nearest neighbors sites (relaxation event). On their turn, these sites can exceed the threshold because of the energy received, and transfer their energy on nearest neighbors sites, and so on. This process is called an avalanche and it is followed until a stable state is reached. After the avalanche stops, the update continues with the deposition and extraction of a new energy unit.

We impose periodic boundary condition on the system, i.e., $e(i, 0) \equiv e(i, L+1)$ and $e(0, j) \equiv e(L+1, j)$. In the usual sandpile this would lead to troubles because $E$ can only increase. Thus after a finite time a state with an infinite avalanche that goes on forever is reached. This state obviously is not related with the critical stationary one. For this reason periodic boundary conditions have never been used to determine the critical properties of sandpile models. The price to pay for that is the inclusion of the strong finite size corrections induced by the finite boundary dissipation.

In the CS and RS models the energy dissipation is acting as an independent driving, while in the usual sandpile it is always linked to the toppling event itself. In SOC sandpile also the average energy is dependent upon driving and dissipation because of the energy balance, while in our microcanonical model this self-organization is ruled out. Thus in these models, the total energy $E$ is a free parameter, that can be freely tuned. Here we will mainly present the CS model and some evidences for an analogous behavior of the RS discrete model, where the critical energy density turns out to coincide with the stationary energy density of the BTW model.

We study the CS sandpile model as a function of the control parameter $E$ : we start with small $E$ (few energy) and small correlation length, and we increase $E$, keeping the correlation length smaller that the lattice size in order to make finite size effects small (we present here only results that do not change when going from $L=256$ to $L=512$ ). We can characterize the system's activity by defining the probability $P_{s}(s)$ that an avalanche is constituted by $s$ relaxation events. In the same way we can study the probability distributions $P_{t}(t)$ and $P_{s_{d}}\left(s_{d}\right)$ which refer to the avalanche time duration and number of distinct sites involved, respectively. When $E \rightarrow E_{c}$ the average avalanche size $\langle s\rangle$ diverges, together with the average avalanche duration $\langle t\rangle$ and the average number of different sites touched during an avalanche, $\left\langle s_{d}\right\rangle$. The system reaches in this case a critical point, and we have determined numerically the probability distributions $P_{s}(s)$, $P_{t}(t)$, and $P_{s_{d}}\left(s_{d}\right)$ as well as the exponents of their asymptotic power law decay.

In Fig. 1 we show the avalanche average size, $\langle s\rangle$, for $L=512$, as a function of $E$, together with the best fit to a simple power divergence (done by using all the points plotted in the figure). We fit the asymptotic behavior:

$$
\langle s\rangle \sim \frac{1}{\left(E_{c}-E\right)^{\gamma}},
$$

and we find $E_{c}=2.596 \pm 0.001$ and $\gamma=1.41 \pm 0.03$ [12]. The average avalanche size can be shown to scale asymptotically as the system response function $\chi_{E}$, that implies $\chi_{E} \sim\left(E_{c}-E\right)^{-\gamma}$. The latter expression characterizes how the system reacts to external perturbations [7].

For the energy range where $\langle s\rangle>20$ we have computed an effective, energy dependent power exponent for the avalanche distribution. We show in Fig. 2 the typical situation (for $L=512$, at $E=2.586$ ): since we are not at $E_{c}$ the power law decay is truncated (at a value that turns out to be of order $\langle s\rangle$ ). We always fit the power law, $P_{s}(s) \sim s^{-\tau_{s}}$ in a range of $s$ that goes from 1 to $\langle s\rangle^{(L)}$. One sees from the figure that the fit (the dashed straight

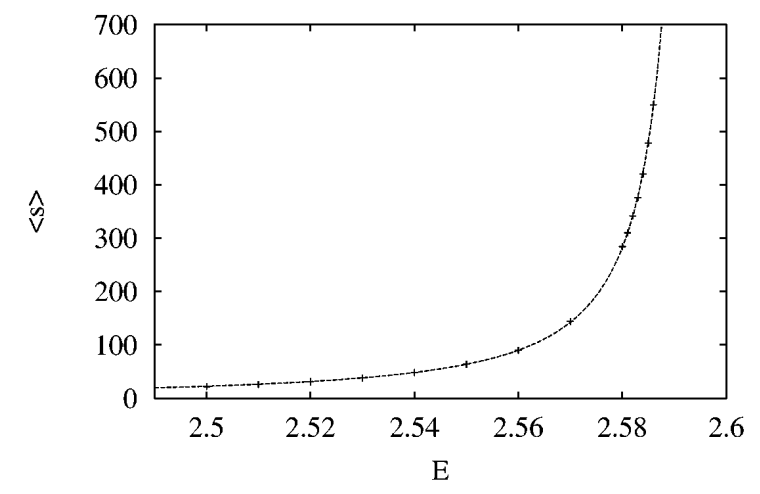

FIG. 1. $\langle s\rangle$ versus $E$, with the best fit to a power divergence. 


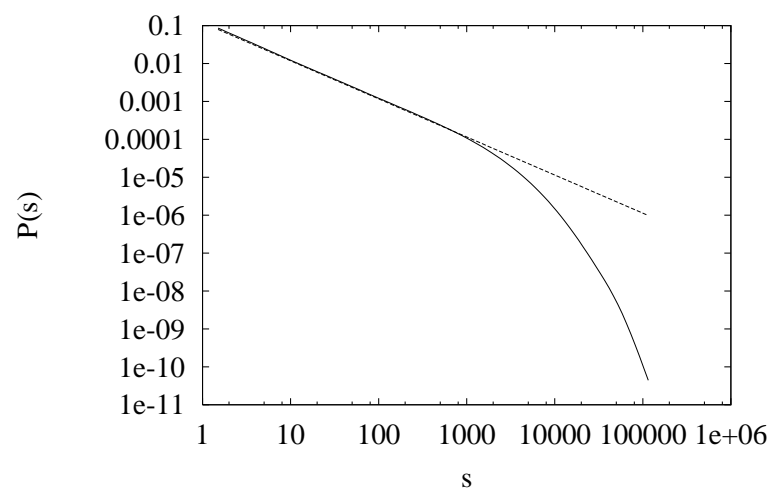

FIG. 2. $P_{s}(s)$ versus $s$ in $\log$-log scale (the solid line is a smooth interpolation of the numerical data) and best power fit (see text).

line) is very good on three decades (the solid line is a smooth interpolation to the numerical data).

The exponents one finds at finite $\left(E_{c}-E\right)$ have to be extrapolated to the critical point. We fit $\tau_{s}$ to an asymptotic value with corrections linear in $\log \left(E_{c}-\right.$ $E)^{-1}$ (by following Manna [13]): we find $\tau_{s}\left(E_{c}\right)=$ $1.26 \pm 0.02$, where again the error is only statistical. Still, in the limit of such a statistical accuracy (that is of the same level it can be reached for the BTW model), we find the same exponent that is believed to describe the BTW scaling. The same procedure works for the time duration of an avalanche. Here by assuming that

$$
\langle t\rangle \sim \frac{1}{\left(E_{c}-E\right)^{\theta}},
$$

we find a very good best fit with $E_{c}=2.597 \pm 0.001$ and $\theta=0.80 \pm 0.04$. With the same approach used for $P_{s}(s)$ we find that $P_{t}(t) \sim t^{-\tau_{t}}$, where $\tau_{t}\left(E_{c}\right)=1.49 \pm 0.04$. It is worth remarking that in measuring the time duration of an avalanche different definitions of time can be used. Here we adopt the one commonly implemented in SOC automata: at each integer time step all currently active sites topple. Again, in the error bars given by the fitting procedure, we find a remarkable agreement with the $\tau_{t}=\frac{3}{2}$ that one expects for the BTW model. The same procedure applied to the different sites touched from an avalanche, $\left\langle s_{d}\right\rangle$, leads again to a divergence at $E_{c}=2.597$ with an exponent equal to $1.34 \pm 0.03$. Again, $P_{s_{d}}\left(s_{d}\right)$ shows a clear power law behavior, and we find that $\tau_{s_{d}}\left(E_{c}\right)=1.27 \pm 0.04$, again in good agreement with the BTW result.

We can also define a natural characteristic length in the system. In general, close to the critical point the avalanche distribution has the scaling form

$$
P(s)=s^{-\tau} G\left(\frac{s}{s_{c}}\right),
$$

where $G(x)$ is a universal function and $s_{c}$ is the avalanche cutoff size. The latter is the system char- acteristic length that close to the critical point scales as $s_{c} \sim\left(E_{c}-E\right)^{-1 / \sigma}$. In order to test the scaling assumption and find an estimate of the $\sigma$ exponent we have used a data collapse technique. For energy values close to the critical one, the plot of $P(s) /\left(E_{c}-E\right)^{\tau / \sigma}$ as a function of the rescaled variable $s /\left(E_{c}-E\right)^{-1 / \sigma}$ must collapse into the same universal curve by using the correct values of $\tau$ and $\sigma$. In Fig. 3 we show the data collapse from avalanche distributions obtained with $\left(E_{c}-E\right)$ ranging over almost 1 order of magnitude. The values we obtain for the exponents are $\tau=1.20 \pm 0.05$ and $\sigma=0.55 \pm 0.03$. By using Eq. (3), we can immediately write the relation

$$
\langle s\rangle=\int s^{-\tau+1} P(s) d s \sim\left(E_{c}-E\right)^{(\tau-2) / \sigma},
$$

which immediately gives the scaling relation $\gamma=(2-$ $\tau) / \sigma$. The latter relation is satisfied by the exponent values we obtain, providing a further consistency check for the numerical results. In the usual sandpile models, a stationary state is reached only if we allow the system to dissipate energy through its boundary or an effective bulk dissipation. Characteristic lengths and scaling exponents can thus be defined with respect to the effective dissipation or boundary length and actually measured in numerical simulations [7]. It is interesting to remark that the values of critical exponents obtained from numerical simulations of the usual sandpiles $(\gamma=1, \sigma=0.77$ [14]) are very different from those obtained in the present paper.

From the previous analysis we can therefore identify two main dynamical mechanisms in SOC models. The first is the self-organization that is driven by the energy balance condition. The sandpile evolves in order to set its energy density so that the avalanche finds a background that allows it to dissipate enough energy. This process does not imply criticality. The second mechanism is the

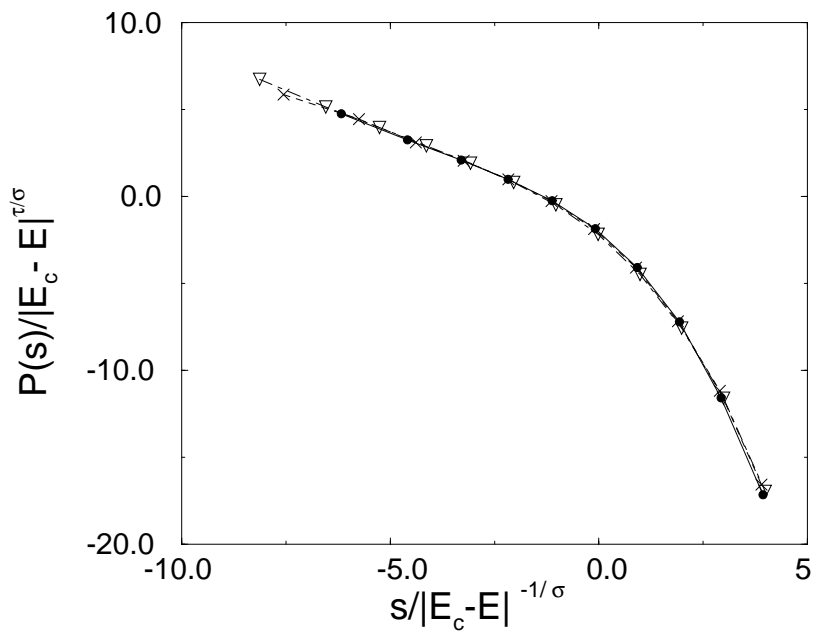

FIG. 3. Scaling plot of $P(s) /\left(E_{c}-E\right)^{\tau / \sigma}$ versus $s / \mid E_{c}-$ $\left.E\right|^{-1 / \sigma}$ in a log-log scale. For the sake of clarity we report also binned data points on top of the full data curves. 
dynamical interaction which builds up in the system the long range correlations which on its turn create the critical avalanche distribution. This is just in the presence of the locality breaking obtained in the limit of infinite slow driving of the system. In our microcanonical version of the model, we control the energy self-organization from outside. The critical point is thus reached just in the presence of the critical energy density which allows the slow driving to generate the critical configuration for the system. These two different ways of reaching the critical point appear to generate different scaling properties with respect to the control parameter. On the contrary the scaling behavior properties right at criticality result in being the same, within the numerical accuracy of our simulations, in both SOC and energy constrained sandpiles automata. With the SOC sandpile and the present model being in different universality classes, this latter result appears to be very puzzling. In this case, only the presence of strong internal symmetries could give rise to an invariant $\tau$ exponent. This makes it also worthwhile to investigate if the model retains this symmetry in higher Euclidean dimensions. On the other hand, larger size simulations are needed in order to definitely rule out finite size bias in the exponents evaluation.

Finally, we report that the RS model shows the same kind of behavior. It is crucial to note that here we find $E_{c}=2.127 \pm 0.004$ to be compared with the $E_{c}=$ 2.125 that Grassberger and Manna [15] find for BTW. The energy where the model becomes critical is exactly the energy reached from BTW in the steady state. This is because the RS model has a microscopic dynamics which is identical to the SOC BTW model. The difference is in the way the system is driven to criticality and thus in the energy constraint. This allows us to compare directly nonuniversal quantities right at the critical point, that should assume the same values in both models. The critical behavior of this model is characterized by the same critical exponents of the CS model. This seems to support the idea that the CS and RS models belong to the same universality class. Thus, the homogeneous dissipation does not introduce any relevant difference with respect to the fully stochastic RS model. The detailed presentation of the RS model numerical data will appear in a forthcoming paper [16].

We are grateful to R. Dickman and S. Zapperi for useful discussions and a careful reading of the manuscript. The main part of the numerical simulations have been run on the Kalix parallel computer [17] (a Beowulf project at
Cagliari Physics Department). We thank Gianni Mula for leading the effort toward organizing this computer facility.

[1] P. Bak, C. Tang, and K. Wiesenfeld, Phys. Rev. Lett. 59, 381 (1987); Phys. Rev. A 38, 364 (1988).

[2] For a review, see G. Grinstein, in Scale Invariance, Interfaces and Non-Equilibrium Dynamics, edited by A. McKane et al., NATO Advanced Study Institutes, Ser. B, Vol. 344 (Plenum, New York, 1995).

[3] G. Durin, G. Bertotti, and A. Magni, Fractals 3, 351 (1995); D. Spasojević, S. Bukvić, S. Milosević, and H.E. Stanley, Phys. Rev. E 54, 2531 (1996), and references therein.

[4] A. Petri, G. Paparo, A. Vespignani, A. Alippi, and M. Costantini, Phys. Rev. Lett. 73, 3423 (1994); S. Zapperi, A. Vespignani, and H. E. Stanley, Nature (London) 388, 658 (1997).

[5] G. Gutenberg and C.F. Richter, Ann. Geophys. 9, 1 (1956).

[6] D. Sornette, A. Johansen, and I. Dornic, J. Phys. I (France) 5, 325 (1995)

[7] A. Vespignani and S. Zapperi, Phys. Rev. Lett. 78, 4793 (1997).

[8] Sandpile models, for instance, are driven by adding a single energy grain on a randomly chosen site, when no active sites are present. In this way, avalanches are instantaneous with respect to the driving time scale. Nonlocality is thus implicitly enforced in computer simulations, where the evolution of a single site depends on the state of the entire system.

[9] R. Dickman, in Nonequilibrium Statistical Mechanics in One Dimension, edited by V. Privman (Cambridge Press, Cambridge, 1996); R. Dickman, A. Vespignani, and S. Zapperi (to be published).

[10] A. Ben-Hur and O. Biham, Phys. Rev. E 53, R1317 (1996).

[11] S. Lübeck and K. D. Usadel, Phys. Rev. E 55, 4095 (1997); S. Lübeck, Phys. Rev. E 56, 1590 (1997).

[12] The error we report is the statistical one obtained from the fitting procedure. The true uncertainty on the result is also affected by the systematic error due to the deviation from scaling, which, however, is difficult to estimate.

[13] S. S. Manna, J. Stat. Phys. 59, 509 (1990); Physica (Amsterdam) 179A, 249 (1991).

[14] A. Chessa, E. Marinari, A. Vespignani, and S. Zapperi, cond-mat/9802123, 1998.

[15] P. Grassberger and S. S. Manna, J. Phys. (Paris) 51, 1077 (1990).

[16] A. Chessa, E. Marinari, and A. Vespignani (to be published).

[17] For information, see the electronic site: http://kalix.dsf. unica.it/ 